\title{
CAMP Gene Promoter Methylation Induces Chondrocyte Apoptosis By Inhibiting ROS Levels And Inflammatory Response
}

\section{Guoliang Wang}

First Affilliated Hospital of Kunming Medical University

\section{Yanlin Li}

First Affiliated Hospital of Kunming Medical University

\section{Guang Yang}

First Affiliated Hospital of Kunming Medical University

\section{Tengyun Yang}

First Affiliated Hospital of Kunming Medical University

\section{Lu He}

First Affiliated Hospital of Kunming Medical University

\section{Yang Wang ( $358556760 @ q q . c o m$ )}

First Affiliated Hospital of Kunming Medical University https://orcid.org/0000-0002-5103-3078

\section{Primary research}

Keywords: CAMP, methylation, osteoarthritis, ROS, Inflammatory factors

Posted Date: January 5th, 2021

DOl: https://doi.org/10.21203/rs.3.rs-139089/v1

License: (c) (1) This work is licensed under a Creative Commons Attribution 4.0 International License.

Read Full License

Version of Record: A version of this preprint was published at Human Genomics on April 23rd, 2021. See the published version at https://doi.org/10.1186/s40246-021-00321-8. 


\section{Abstract}

Objective: The occurrence of osteoarthritis is related to genetic and environmental factors. Among them, the change of chondrocyte gene expression pattern regulated by epigenetic modification is an important participant. This study analyzed the effect of CAMP gene methylation on the level of oxidative stress and inflammation of chondrocytes.

Methods: We analyzed the changes of the transcriptome in the articular cartilage tissue of osteoarthritis patients (OA) from the GSE117999 dataset. The GSE48422 dataset was used to analyze the changes in the methylation level of osteoarthritis cells. MTT assay and flow cytometry analysis of short hairpin RNA (shRNA) silencing CAMP gene and 5 $\mathrm{M}$ 5-Aza-2'-Deoxycytidine (AZA) treatment on the proliferation and apoptosis of Human Chondrocytes Osteoarthritis (HC-OA) cells. The DCFH-DA assay was used to detect the level of reactive oxygen species (ROS), and the expression level of inflammatory factors was analyzed by Western Blot.

Results: The expression of CAMP in cartilage tissue of OA patients was up-regulated, and the level of methylation was down-regulated. CAMP was highly expressed in osteoarthritis articular cartilage cells. Silencing CAMP inhibited the proliferation of HC-OA cells and promoted their apoptosis. CAMPgene methylation inhibited ROS levels and TNF- $\alpha$ expression levels in HC-OA cells, and promoted TGF- $\beta$ expression. CAMP gene methylation inhibited the proliferation of $\mathrm{HC}-\mathrm{OA}$ cells and promoted their apoptosis.

Conclusion: $C A M P$ gene promoter methylation induces chondrocyte apoptosis by inhibiting ROS levels and inflammation.

\section{Introduction}

Osteoarthritis $(\mathrm{OA})$ is a common age-related degenerative disease. Its main pathological feature is articular cartilage degeneration, which is mainly manifested by the loss of articular cartilage, the formation of osteophytes, subchondral bone sclerosis and synovium inflammation, etc., clinical symptoms such as pain, joint deformity and dysfunction may appear in the later clinical stage[1-3].

Research in recent years has shown that epigenetic modification plays an important role in the pathogenesis of $\mathrm{OA}[4,5]$. Epigenetic modification mainly includes DNA methylation, histone modification, chromatin remodeling and non-coding RNA, among which DNA methylation is closely related to inflammatory diseases[6, 7]. At present, DNA methylation studies of OA mainly focus on articular cartilage, because articular cartilage is the core tissue involved in the disease process[8]. In addition, DNA methylation has become an important regulator of chondrocyte dedifferentiation, severely destroying the results of autologous chondrocyte implantation in patients with cartilage injury[8]. Therefore, it is of great significance to study the DNA methylation profile of chondrocyte dedifferentiation. 
At present, the methylation sites or regions identified in OA genome-wide methylation studies are mostly enriched in genes related to immunity or inflammation, cartilage development, transcription factor regulation, and protease activity $[9,10]$. Although OA was once considered a non-inflammatory disease, it has been confirmed that inflammation is very important in the occurrence of $O A[11]$.

Tumor necrosis factor a (TNF-a) is one of the main pro-inflammatory cytokines related to the pathogenesis of OA, it can stimulate the release of matrix metalloenase-1 (MMP-1), MMP-3 and MMP-13 in OA patients, and inhibit the synthesis of proteoglycans and type II collagen[12]. Transforming growth factor $\beta$ (TGF- $\beta$ ) is a member of the TGF- $\beta$ superfamily. It transmits signals to the nucleus through the TGF- $\beta$ receptor and its intracellular signaling system to play a variety of different physiological regulatory roles $[13,14]$. Studies have shown that TGF- $\beta$ plays an important role in maintaining normal articular cartilage and joint repair[15].

Some researchers have used the demethylating agent AZA for in vitro intervention of OA chondrocytes. For example, lliopoulos et al.[16] found that AZA could reduce the methylation level of the leptin gene promoter of chondrocytes and increased the expression of leptin mRNA, which then activated matrix metallopeptidase 13 (MMP-13). Kim et al.[17] reported that when OA articular chondrocytes were cocultured with $10 \mu \mathrm{M}$ AZA for 8 days, the methylation level of $6 \mathrm{CpG}$ islands in the SOX-9 promoter region of chondrocytes decreased and the expression level of SOX-9 increased.

The CAMP gene (also known as $L L-37$ ) is located on chromosome 3p21.31. The CAMP protein is composed of 37 amino acids in a spiral shape and has a broad-spectrum antibacterial activity. Studies have shown that CAMP has defensive effects, such as regulating inflammation, inducing immune cells to injured or infected sites, binding and neutralizing Lipopolysaccharide (LPS), promoting epithelialization and repairing the damage[18]. Some researchers had found that CAMP affected the expression and distribution of TLR in tissue mast cells[19]. CAMP can be used as a powerful inducer of CCL3 and ROS generation[19]. Research by Li et al.[20] showed that CAMP could promote epithelial and smooth musclelike differentiation of adipose stem cells through Wnt/ $\beta$-Catenin and NF-KB pathways.

In this study, we analyzed CAMP expression and methylation levels in OA chondrocytes through the GSE117999 dataset and GSE48422 dataset, and analyzed the effects of CAMP methylation on chondrocyte oxidative stress and inflammation level in vitro, as well as the impact on chondrocyte proliferation and apoptosis. It is of great significance for further elucidating the mechanism of $O A$, and it also provides a basis for early diagnosis and treatment of OA.

\section{Materials And Methods}

\subsection{Datasets}

Both the GSE117999 dataset and GSE48422 dataset[21] can be obtained from the Gene Expression Omnibus (https://www.ncbi.nlm.nih.gov/geo/) database. The GSE117999 dataset The GSE117999 
dataset contains transcript data from OA and non-OA cartilage tissue. The GSE48422 dataset contains gene methylation levels in OA patients and non-OA cartilage tissues.

\subsection{Cell culture}

Human Chondrocytes Osteoarthritis (HC-OA) cells were purchased from Cell Applications (San Diego, California, USA), and Human Chondrocytes-articular (HC-A) cells were purchased from ScienCell (San Diego, California, USA). HC-OA cells and HC-A cells were cultured in supplemented with $10 \%$ fetal bovine serum (FBS) (Sigma-Aldrich, St. Louis, MO, USA), 1\% penicillin-streptomycin (ThermoFisher Scientific, Waltham, MA) Chondrocyte growth medium (PromoCell, Heidelberg, Germany) at $37^{\circ} \mathrm{C}$ in a humidified incubator containing $5 \% \mathrm{CO}_{2}$.

\subsection{Cell transfection and 5-Aza-2'-Deoxycytidine (AZA) treatment}

In order to study the effect of CAMP on the proliferation and apoptosis of chondrocytes, we used small hairpin RNA to silence CAMP gene expression (sh-CAMP), no template control(sh-NC) and no transfection group (Control) as controls. According to the manufacturer's instructions, HC-OA cells $(5 \times 105$ cells/well) were transfected with Lipofectamine 3000 (Life Technologies, Gaithersburg, MD, USA) and incubated in a medium containing 10\% FBS for 48 hours. The detailed process of AZA processing has been previously reported[22]. Quantitative reverse transcription-PCR (qRT-PCR) and Western blot were used to detect transfection efficiency.

\subsection{Cell Counting Kit-8 assay}

Cell Counting Kit-8 (CCK-8) assay was used to detect HC-OA cells proliferation ability. HC-OA cells were seeded in 96 -well plates $\left(1.0 \times 10^{4}\right.$ cells/well), incubated at $37^{\circ} \mathrm{C}$ for $0 \mathrm{~h}, 24 \mathrm{~h}, 48 \mathrm{~h}, 72 \mathrm{~h}$, and then added to CCK-8 (Beyotime, Haimen, China) and incubated for 2 h. A microplate reader (BIOTEK, Vermont, USA) was used to detect the optical density (OD) value at $450 \mathrm{~nm}$.

\subsection{ROS level detection}

The cultured HC-OA cells were washed with $1 \times$ phosphate buffered solution (PBS), and the Reactive Oxygen Species Assay Kit (Sigma-Aldrich, Minneapolis, USA) was used to detect ROS levels according to the supplier's instructions. Three replicate wells in each group, a microplate reader (BIOTEK, Vermont, USA) was used to detect the absorbance at 485/535 $\mathrm{nm}$.

\subsection{Quantitative reverse transcription-PCR (qRT-PCR)}

The total RNA in HC-OA cell was extracted by TRIzol reagent (Invitrogen, Carlsbad, CA). The concentration of the extracted RNA was determined by NanoDrop 2000 (Thermo Fisher Scientific). Reverse transcription kit (Takara, Dalian, China) was used to reverse transcribe the extracted total RNA into CDNA. QRT-PCR was performed on an Applied Biosystems StepOnePlus Real-time PCR system (Applied Biosystems, Foster City, CA, USA) using SYBR Green Real-time PCR Master Mix (Toyobo, Osaka, Japan) according to the manufacturer's protocol. GAPDH were served as endogenous controls. The CAMP mRNA primers 
sequence: 5'-GAA GAC CCA AAG GAA TGG CC-3'(forword), 5'-TCA GAG CCC AGA AGC CTG AG-3'(reverse). GAPDH primers sequence: 5'-GAA GGT GAA GGT CGG AGT C-3'(forword), 5'-GAA GAT GGT GAT GGG ATT TC-3'(reverse). The relative expression of CAMP mRNA was calculated using the $2^{-\Delta \Delta C t}$ method.

\subsection{Western Blot}

HC-OA cells were lysed with RIPA buffer (Beyotime, Shanghai, China) for half an hour, and centrifuged at $17000 \mathrm{~g}$ for $45 \mathrm{~min}$ at $4^{\circ} \mathrm{C}$. BCA protein assay kit (Beyotime, Shanghai, China) was used to detect protein concentration. Then sodium dodecyl sulfate-polyacrylamide gel electrophoresis (SDS-PAGE) was used to separate the proteins, and proteins were transferred to polyvinylidene fluoride (PVDF) membrane, and then use Tween-Tris buffered saline (TTBS) containing 5\% skim milk to seal the PVDF membrane at room temperature. Then, anti-CAMP (\#ab80895, 1:1000, Abcam, Cambridge, UK) and anti-GAPDH (\#ab181602, 1:1000, Abcam, Cambridge, UK) were incubated with PVDF membrane overnight at $4{ }^{\circ} \mathrm{C}$. The membrane was then washed 3 times with TTBS, and incubated with a secondary antibody conjugated with horseradish peroxidase at room temperature for 2 hours. The blot was visualized with an enhanced chemiluminescence (ECL) kit (Santa Cruz Biotechnology) and scanned by Chemlmager 5500 V2.03 software. The relative integrated density value (IDV) was calculated using GAPDH as an internal control.

\subsection{Statistical analysis}

GraphPad Prism 8.0 (GraphPad Software, San Diego, CA) was used for statistical analysis of the data in this study. Two-tailed Student's t test and analysis of variance (ANOVA) were used to the difference between groups. $p<0.05$ was considered to have statistical significance.

\section{Results}

\subsection{The expression of CAMP in cartilage tissue of OA patients was up-regulated, and the level of methylation was down-regulated}

The analysis of the GSE117999 data set shows that CAMP was up-regulated in the articular cartilage of patients with OA (Table 1, Fig. 1a). The analysis of the GSE48422 dataset[21] showed that the level of CAMP methylation was down-regulated (Table 2, Fig. 1b). 
Table 1

Top 15 up-regulated and down-regulated gene in arthritic samples from patients with and without osteoarthritis ranked by logFC after GEO2R analysis.

\begin{tabular}{|c|c|c|c|}
\hline GENE_SYMBOL & adj.P.Val & $\log F C$ & Up/Down \\
\hline CRB1 & $1.52 \mathrm{E}-04$ & 1.01580843 & Up \\
\hline ZSCAN12 & 1.89E-03 & 1.0271285 & Up \\
\hline LE01 & $1.21 \mathrm{E}-04$ & 1.03342688 & Up \\
\hline ESR1 & 4.03E-04 & 1.04997982 & Up \\
\hline FAM20A & $2.36 \mathrm{E}-03$ & 1.05411929 & Up \\
\hline ZNF354B & $2.06 \mathrm{E}-04$ & 1.05630409 & Up \\
\hline CCR10 & $1.15 \mathrm{E}-03$ & 1.06099425 & Up \\
\hline INCENP & $1.21 \mathrm{E}-04$ & 1.18121011 & Up \\
\hline CAMP & $3.24 \mathrm{E}-03$ & 1.23685287 & Up \\
\hline THAP5 & $1.31 \mathrm{E}-05$ & 1.27811954 & Up \\
\hline SMNDC1 & 8.17E-04 & 1.29092563 & Up \\
\hline HOXC8 & $9.69 \mathrm{E}-05$ & 1.30931826 & Up \\
\hline LCE2B & 3.73E-04 & 1.34166775 & Up \\
\hline FAM90A1 & 2.87E-03 & 1.40391379 & Up \\
\hline QRFPR & $4.59 \mathrm{E}-04$ & 1.57552192 & Up \\
\hline SLC38A6 & $1.41 \mathrm{E}-03$ & -1.64465134 & Down \\
\hline PCDHB10 & $6.24 \mathrm{E}-04$ & -1.43430506 & Down \\
\hline HLA-DRB4 & $1.21 \mathrm{E}-04$ & -1.4061153 & Down \\
\hline TOLLIP & 8.09E-04 & -1.31133046 & Down \\
\hline HLA-DQA2 & $9.20 \mathrm{E}-04$ & -1.29212829 & Down \\
\hline TP53TG3D & 1.99E-03 & -1.25442713 & Down \\
\hline CHST1 & $2.18 \mathrm{E}-03$ & -1.1748763 & Down \\
\hline ANO8 & $2.34 \mathrm{E}-03$ & -1.16784996 & Down \\
\hline TRIM78P & $2.18 \mathrm{E}-03$ & -1.10578964 & Down \\
\hline ADHFE1 & 1.66E-03 & -1.07972333 & Down \\
\hline
\end{tabular}




\begin{tabular}{|llll|}
\hline GENE_SYMBOL & adj.P.Val & logFC & Up/Down \\
\hline DUT & $3.48 E-03$ & -1.07647613 & Down \\
\hline SS18L1 & $2.18 \mathrm{E}-03$ & -1.07511907 & Down \\
\hline FRMD8 & $1.72 \mathrm{E}-04$ & -1.06645789 & Down \\
\hline FAM184B & $2.18 \mathrm{E}-03$ & -1.06047291 & Down \\
\hline TMEM259 & $9.41 \mathrm{E}-04$ & -1.05326043 & Down \\
\hline FC, fold change. & & & \\
\hline
\end{tabular}


Table 2

Top 20 hypomethylated genes gene in arthritic and non-arthritic knee cartilage samples ranked by logFC after GEO2R analysis.

\begin{tabular}{|llll|}
\hline GENE_SYMBOL & P.Value & logFC & Up/Down \\
\hline GUCY2D & 0.00902988 & 4.21533489 & Up \\
\hline GPR23 & 0.01044718 & 4.10888772 & Up \\
\hline LMBR1L & 0.0050466 & 4.06301737 & Up \\
\hline MOBP & 0.02433125 & 3.81893625 & Up \\
\hline RASSF8 & 0.00004736 & 3.71186833 & Up \\
\hline SLC6A5 & 0.02136651 & 3.49741335 & Up \\
\hline ING4 & 0.00371036 & 3.40976791 & Up \\
\hline CDK5 & 0.01690306 & 3.39845271 & Up \\
\hline hsa-mir-29b-2 & 0.00810529 & 3.39799728 & Up \\
\hline PITX1 & 0.03355549 & 3.31112165 & Up \\
\hline TLE6 & 0.02379696 & 3.31057234 & Up \\
\hline IL22 & 0.02971686 & 3.29164562 & Up \\
\hline CDIPT & 0.02299476 & 3.24065781 & Up \\
\hline FLJ10986 & 0.0034974 & 3.21953344 & Up \\
\hline KIAA1970 & 0.02280297 & 3.1642642 & Up \\
\hline GDF7 & 0.01267419 & 3.14401884 & Up \\
\hline CAB39L & 0.00421281 & 3.13792295 & Up \\
\hline CHAF1B & 0.00197712 & 3.13636909 & Up \\
\hline C15orf2 & 0.01644456 & 3.07462778 & Up \\
\hline TMPRSS6 & 0.04829902 & 3.06043658 & Up \\
\hline NDUFV3 & 0.01618486 & -3.45593987 & Down \\
\hline CINP & 0.01970577 & -3.40575442 & Down \\
\hline ACOX3 & 0.0123907 & -3.09168387 & Down \\
\hline KLK15 & 0.00245482 & -3.01407804 & Down \\
\hline RP13-360B22.2 & 0.04529023 & -2.9067811 & Down \\
\hline
\end{tabular}

FC, fold change. 


\begin{tabular}{|llll|}
\hline GENE_SYMBOL & P.Value & logFC & Up/Down \\
\hline C10orf120 & 0.04175249 & -2.85198326 & Down \\
\hline C10orf26 & 0.03356837 & -2.83509207 & Down \\
\hline ATP6AP2 & 0.00771311 & -2.81820993 & Down \\
\hline MED28 & 0.01218592 & -2.77282343 & Down \\
\hline ZDHHC16 & 0.0367154 & -2.75390711 & Down \\
\hline LOC57228 & 0.03643365 & -2.71534123 & Down \\
\hline C15orf5 & 0.00278977 & -2.67864013 & Down \\
\hline ALDH1A3 & 0.03431337 & -2.65248752 & Down \\
\hline DNMT2 & 0.03566104 & -2.64961214 & Down \\
\hline MGC41945 & 0.02997186 & -2.64282551 & Down \\
\hline ASF1B & 0.0193366 & -2.62752981 & Down \\
\hline CAMP & 0.01873345 & -2.62260485 & Down \\
\hline ZNF44 & 0.01006365 & -2.56469726 & Down \\
\hline IDS & 0.00377758 & -2.56033268 & Down \\
\hline TCN2 & 0.01701305 & -2.53741851 & Down \\
\hline FC, fold change. & & & \\
\hline & & -2 & \\
\hline
\end{tabular}

\subsection{CAMP was highly expressed in osteoarthritis articular cartilage cells}

We analyzed the expression levels of CAMP in the cartilage tissue of 8 cases of OA patients undergoing total knee arthroplasty and 5 cases of trauma requiring high amputation (excluding $\mathrm{OA}$ ) (Control). The results showed that the expression levels of $C A M P$ mRNA and CAMP protein in the articular cartilage tissue of KOA patients were significantly higher than those of the Control group $(p=0.002, p<0.0001$, Fig. 2a, 2b). Our analysis in Human Chondrocytes Osteoarthritis (HC-OA) cells and Human Chondrocytesarticular ( $\mathrm{HC}-\mathrm{A})$ cells showed that the CAMP mRNA level and CAMP protein level in HC-OA cells were significantly higher than those in HC-A cells $(p<0.001, p<0.0001$, Fig. $2 \mathrm{c}, 2 \mathrm{~d})$.

\subsection{Silencing CAMP gene inhibited the proliferation of osteoarthritis articular cartilage cells and promoted their apoptosis}


In order to study the role of CAMP gene in the proliferation and apoptosis of chondrocytes in OA patients, we used small hairpin RNA to silence CAMP gene (sh-CAMP), no template control (sh-NC) and no transfection group (Control) as control. QRT-PCR and Western Blot results confirmed that CAMP gene was successfully knocked out in HC-OA cells $(p<0.001, p<0.01$; Fig. 3a, 3b). MTT assay results showed that compared with the Control, the proliferation ability of HC-OA cells was significantly inhibited after shCAMP transfection $(p<0.01$, Fig. $3 c)$. Flow cytometry detection of cell apoptosis showed that the apoptotic rate of HC-OA cells after sh-CAMP transfection was significantly higher than that of Control ( $p$ $<0.01$, Fig. 3d).

\subsection{CAMP gene methylation inhibited ROS levels and inflammatory response levels in osteoarthritis articular cartilage cells}

In order to further study the effect of CAMP methylation on the level of oxidative stress and inflammatory response levels in HC-OA cells, we set up 3 groups, namely Control, sh-CAMP transfected HC-OA cell group (sh-CAMP), $5 \mu \mathrm{M}$ 5-Aza-2'-Deoxycytidine (AZA) treatment group. First, Western Blot results showed that compared with the Control, the CAMP protein expression in HC-OA cells was significantly downregulated after sh-CAMP transfection, and CAMP expression in HC-OA cells was significantly increased after AZA treatment (Fig. 4a). After transfection with sh-CAMP, the level of ROS in HC-OA cells decreased significantly, while the level of ROS in HC-OA cells increased after AZA treatment (Fig. 4b). Western Blot results showed that compared with Control, TNF-a levels in HC-OA cells were significantly decreased after sh-CAMP transfection, and TNF-a levels were significantly increased after AZA treatment (Fig. 4c). The level of TGF- $\beta$ in HC-OA cells was significantly increased after sh-CAMP transfection, and AZA treatment down-regulated the expression of TGF- $\beta$ (Fig. 4d). These results showed that CAMP gene methylation down-regulated the expression of CAMP in HC-OA cells and inhibited the level of oxidative stress and inflammatory response levels.

\subsection{CAMP gene methylation inhibited the proliferation of osteoarthritis articular cartilage cells and promoted their apoptosis}

In order to further study the effect of CAMP gene methylation on the proliferation and apoptosis of $\mathrm{HC}-\mathrm{OA}$ cells, we set up 3 groups, namely Control, sh-CAMP, and AZA. Western Blot analysis showed that, compared with Control, the CAMP protein in the sh-CAMP transfection group was significantly downregulated, and the CAMP protein expression level was significantly increased after AZA treatment (Fig. 5a). MTT assay results showed that compared with Control, the proliferation ability of HC-OA cells in the sh-CAMP transfection group was inhibited, and the proliferation ability of HC-OA cells was significantly increased after AZA treatment (Fig. 5b). The results of flow cytometry showed that compared with Control, the apoptotic rate of HC-OA cells in the sh-CAMP transfection group was 
significantly increased, and the apoptotic rate of HC-OA cells was significantly decreased after AZA treatment (Fig. 5c). These results showed that CAMP gene methylation inhibited the proliferation of osteoarthritis articular cartilage cells and promoted their apoptosis.

\section{Discussion}

In this study, we extracted and analyzed the differentially expressed genes and methylation levels in chondrocytes from public databases, and found that CAMP (also known as LL-37) was up-regulated in the articular cartilage of patients with $\mathrm{OA}$, the methylation level of CAMP gene was down-regulated. We found that CAMP protein expression was up-regulated in articular cartilage tissue of OA patients and HC$\mathrm{OA}$ cells. After silencing the expression of the CAMP gene, we found that the proliferation of HC-OA cells was inhibited, accompanied by a significant increase in the apoptotic rate. After demethylation, the level of ROS and inflammation in HC-OA cells increased significantly, the cell proliferation ability was significantly enhanced, and the apoptosis rate was significantly reduced. Therefore, we speculated that CAMP gene promoter methylation induced chondrocyte apoptosis by inhibiting ROS levels and inflammatory response levels.

Current research evidence shows that risk factors for OA include genetics, inflammation, environmental factors, age, stress stimulation, etc.[23, 24]. In recent years, more and more studies have found that epigenetic modification plays an important role in the pathogenesis of OA. Epigenetic modification works by regulating gene transcription or post-transcriptional regulation, including DNA methylation, histone modification, chromosome remodeling and non-coding RNAs (ncRNAs) $[6,25,26]$. Usually DNA methylation occurs in cytosine, guanine, and nucleotides (CpG), which is mainly manifested as the phenomenon of conversion of cytosine to 5-methylcytosine[27, 28]. The research on DNA methylation function mainly focuses on the transcription start site of genes[29]. Methylation of the promoter region can inhibit gene expression, on the one hand, methylation of the $\mathrm{CpG}$ site directly interferes with the binding of transcription factors to DNA in the regulatory region; on the other hand, methylated DNA and methylated $\mathrm{CpG}$ binding region proteins such as MeCP2 specifically bind to form a complex, which restricts the passage of transcription factors to their binding sites, thereby inhibiting gene expression[3032].

In this study, we found that the expression level of CAMP protein increased significantly after AZA treatment. The reason might be that the methylation level of the promoter region of CAMP gene decreased after AZA treatment, and the binding efficiency of CAMP gene transcription factors increased, as a result, the expression level of CAMP increased significantly. At present, researchers have tried to use AZA for in vitro intervention of OA chondrocytes. For example, Alvarez-Garcia et al.[33] treated TC28 cells with AZA and the expression of ATOH8 and TBX4 increased significantly. In addition, some researchers found that the DNA methylation level of the iNOS enhancer-5.8 kb CpG site decreased after AZA treatment, and the iNOS expression level increased, the methylation of the iNOS enhancer could inhibit the cell cycle process by downregulating NF-KB level and reduce the pro-inflammatory response, which had important therapeutic significance for $\mathrm{OA}[34]$. 
In this study, we found that CAMP was highly expressed in articular cartilage cells of OA patients. Silencing the expression of $C A M P$ gene inhibited the proliferation of osteoarthritis articular cartilage cells and promoted their apoptosis, suggesting that CAMP may play an important role in the pathogenesis of osteoarthritis. Yu et al.[35] showed that CAMP inhibited inflammation and promoted bone formation of bone marrow stromal cells (BMSC) through purinergic receptor P2 $\times 7(\mathrm{P} 2 \mathrm{RX} 7)$ and mitogen-activated protein kinase (MAPK) signaling pathway. Further in vitro studies had found that $C A M P$ gene methylation inhibited the level of ROS and TNF- $\alpha$ in chondrocytes, promoted the level of TGF- $\beta$ expression, inhibited the proliferation of chondrocytes, and promoted their apoptosis. The results of Kuensaen et al.[36] found that high levels of CAMP promote the expression of downstream pro-inflammatory cytokines (especially IL17A), which was related to the pathogenesis of inflammatory arthritis. Hu et al.[37] found that CAMP regulated the production of inflammatory cytokines such as TNF-a and inhibited cell apoptosis. The results of this study suggested that the expression of $C A M P$ gene and methylation in promoter region might be involved in the occurrence of osteoarthritis from the level of oxidative stress and the expression of inflammatory factors.

There are some limitations in this study. First of all, the treatment of the articular cartilage tissue samples used for analysis is unclear, and whether there are drugs that affect the expression of inflammatory factors in tissue is unclear. Secondly, the expression and methylation of CAMP gene may lead to the occurrence of OA through other signaling pathways, which was not confirmed in this study. In addition, the results of this study need to be further confirmed in vivo.

\section{Conclusion}

In this study, we found that CAMP gene promoter methylation induces chondrocyte apoptosis by inhibiting ROS levels and inflammatory response levels, which is a potential target for OA therapy.

\section{Declarations}

\section{Ethics approval and consent to participate}

Not applicable

\section{Consent for publication}

Not applicable

\section{Availability of data and materials}

The datasets during and/or analysed during the current study available from the corresponding author on reasonable request.

\section{Competing interests}


The authors declare that they have no competing interests.

\section{Funding}

This work was supported by National Natural Science Foundation of China (No. 81960409, No.81760403), Yunnan Province Clinical Center for Bone and joint Diseases (No. ZX2019-03-04), Yunnan Province Medical Leaders Talent Project (No.L-201601), Expert workstation project of Shiyi Chen [No.2018IC102] and Joint special fund project of applied basic research of Kunming medical university, department of science and technology of Yunnan province (No. 202001AY070001-043).

\section{Authors' contributions}

Yang Wang contributed to the experiment design, manuscript draft, and data analysis. Guoliang Wang and Yanlin Li contributed to the experiment implementation, manuscript draft and data analysis. Guang Yang, Tengyun Yang and Lu He designed and performed the experiments. Guoliang Wang and Yang Wang analyzed the data. Guoliang Wang and Yanlin Li wrote the manuscript. All authors read and approved the final manuscript.

\section{Acknowledgements}

Not applicable

\section{References}

1. Glyn-Jones S, et al. Osteoarthritis Lancet. 2015;386(9991):376-87.

2. Mandl LA. Osteoarthritis year in review 2018: clinical. Osteoarthritis Cartilage. 2019;27(3):359-64.

3. Abramoff B, Caldera FE. Osteoarthritis: Pathology, Diagnosis, and Treatment Options. Med Clin North Am. 2020;104(2):293-311.

4. Shen J, et al. Inflammation and epigenetic regulation in osteoarthritis. Connect Tissue Res. 2017;58(1):49-63.

5. Kim H, et al. Epigenetic Regulation of Chondrocyte Catabolism and Anabolism in Osteoarthritis. Mol Cells. 2015;38(8):677-84.

6. Chen Y, et al. Epigenetic modification of nucleic acids: from basic studies to medical applications. Chem Soc Rev. 2017;46(10):2844-72.

7. Liberman N, Wang SY, Greer EL. Transgenerational epigenetic inheritance: from phenomena to molecular mechanisms. Curr Opin Neurobiol. 2019;59:189-206.

8. Duan L, et al. DNA Methylation Profiling in Chondrocyte Dedifferentiation In Vitro. J Cell Physiol. 2017;232(7):1708-16.

9. Jeffries MA, et al. Genome-wide DNA methylation study identifies significant epigenomic changes in osteoarthritic cartilage. Arthritis Rheumatol. 2014;66(10):2804-15. 
10. Wang W, et al. Genome-wide DNA methylation profiling of articular cartilage reveals significant epigenetic alterations in Kashin-Beck disease and osteoarthritis. Osteoarthritis Cartilage. 2017;25(12):2127-33.

11. Sokolove J, Lepus CM. Role of inflammation in the pathogenesis of osteoarthritis: latest findings and interpretations. Ther Adv Musculoskelet Dis. 2013;5(2):77-94.

12. Kapoor M, et al. Role of proinflammatory cytokines in the pathophysiology of osteoarthritis. Nat Rev Rheumatol. 2011;7(1):33-42.

13. Morikawa M, Derynck R, Miyazono K, TGF-beta and the TGF-beta Family: Context-Dependent Roles in Cell and Tissue Physiology. Cold Spring Harb Perspect Biol, 2016. 8(5).

14. Vander Ark A, Cao J, Li X. TGF-beta receptors: In and beyond TGF-beta signaling. Cell Signal. 2018;52:112-20.

15. Zhen G, et al. Inhibition of TGF-beta signaling in mesenchymal stem cells of subchondral bone attenuates osteoarthritis. Nat Med. 2013;19(6):704-12.

16. Iliopoulos D, Malizos KN, Tsezou A. Epigenetic regulation of leptin affects MMP-13 expression in osteoarthritic chondrocytes: possible molecular target for osteoarthritis therapeutic intervention. Ann Rheum Dis. 2007;66(12):1616-21.

17. Kim KI, Park YS, Im GI. Changes in the epigenetic status of the SOX-9 promoter in human osteoarthritic cartilage. J Bone Miner Res. 2013;28(5):1050-60.

18. Nagaoka I, Tamura H, Reich J. Therapeutic Potential of Cathelicidin Peptide LL-37, an Antimicrobial Agent, in a Murine Sepsis Model. Int J Mol Sci, 2020. 21(17).

19. Agier J, et al. Cathelicidin LL-37 Affects Surface and Intracellular Toll-Like Receptor Expression in Tissue Mast Cells. J Immunol Res. 2018;2018:7357162.

20. Li Y, et al. Cathelicidin LL37 Promotes Epithelial and Smooth-Muscle-Like Differentiation of AdiposeDerived Stem Cells through the Wnt/beta-Catenin and NF-kappaB Pathways. Biochemistry. 2017;82(11):1336-45.

21. Moazedi-Fuerst FC, et al. Epigenetic differences in human cartilage between mild and severe OA. J Orthop Res. 2014;32(12):1636-45.

22. Reynard LN, et al. CpG methylation regulates allelic expression of GDF5 by modulating binding of SP1 and SP3 repressor proteins to the osteoarthritis susceptibility SNP rs143383. Hum Genet. 2014;133(8):1059-73.

23. O'Neill TW, McCabe PS, McBeth J. Update on the epidemiology, risk factors and disease outcomes of osteoarthritis. Best Pract Res Clin Rheumatol. 2018;32(2):312-26.

24. Scheler F, Verwiebe R. [Therapeutic use of diuretics and digitalis glycosides in renal failure]. Verh Dtsch Ges Inn Med. 1989;95:252-60.

25. Skvortsova K, lovino N, Bogdanovic O. Functions and mechanisms of epigenetic inheritance in animals. Nat Rev Mol Cell Biol. 2018;19(12):774-90.

26. Widschwendter M, et al. Epigenetic stem cell signature in cancer. Nat Genet. 2007;39(2):157-8. 
27. Mersakova S, et al. Methylation of CADM1 and MAL together with HPV status in cytological cervical specimens serves an important role in the progression of cervical intraepithelial neoplasia. Oncol Lett. 2018;16(6):7166-74.

28. Liu K, et al. Structural basis for the ability of MBD domains to bind methyl-CG and TG sites in DNA. J Biol Chem. 2018;293(19):7344-54.

29. Jones PA. Functions of DNA methylation: islands, start sites, gene bodies and beyond. Nat Rev Genet. 2012;13(7):484-92.

30. Moore LD, Le T, Fan G. DNA methylation its basic function Neuropsychopharmacology. 2013;38(1):23-38.

31. Clemens AW, et al. MeCP2 Represses Enhancers through Chromosome Topology-Associated DNA Methylation. Mol Cell. 2020;77(2):279-93 e8.

32. Kinde B, et al. DNA methylation in the gene body influences MeCP2-mediated gene repression. Proc Natl Acad Sci U S A. 2016;113(52):15114-9.

33. Alvarez-Garcia O, et al. Increased DNA Methylation and Reduced Expression of Transcription Factors in Human Osteoarthritis Cartilage. Arthritis Rheumatol. 2016;68(8):1876-86.

34. de Andres MC, Takahashi A, Oreffo RO. Demethylation of an NF-kappaB enhancer element orchestrates iNOS induction in osteoarthritis and is associated with altered chondrocyte cell cycle. Osteoarthritis Cartilage. 2016;24(11):1951-60.

35. Yu X, et al. LL-37 inhibits LPS-induced inflammation and stimulates the osteogenic differentiation of BMSCs via P2 × 7 receptor and MAPK signaling pathway. Exp Cell Res. 2018;372(2):178-87.

36. Kuensaen C, et al. LL-37 alone and in combination with IL17A enhances proinflammatory cytokine expression in parallel with hyaluronan metabolism in human synovial sarcoma cell line SW982-A step toward understanding the development of inflammatory arthritis. PLoS One. 2019;14(7):e0218736.

37. Hu Z, et al. Antimicrobial cathelicidin peptide LL-37 inhibits the pyroptosis of macrophages and improves the survival of polybacterial septic mice. Int Immunol. 2016;28(5):245-53.

\section{Figures}


$\mathbf{a}$

b
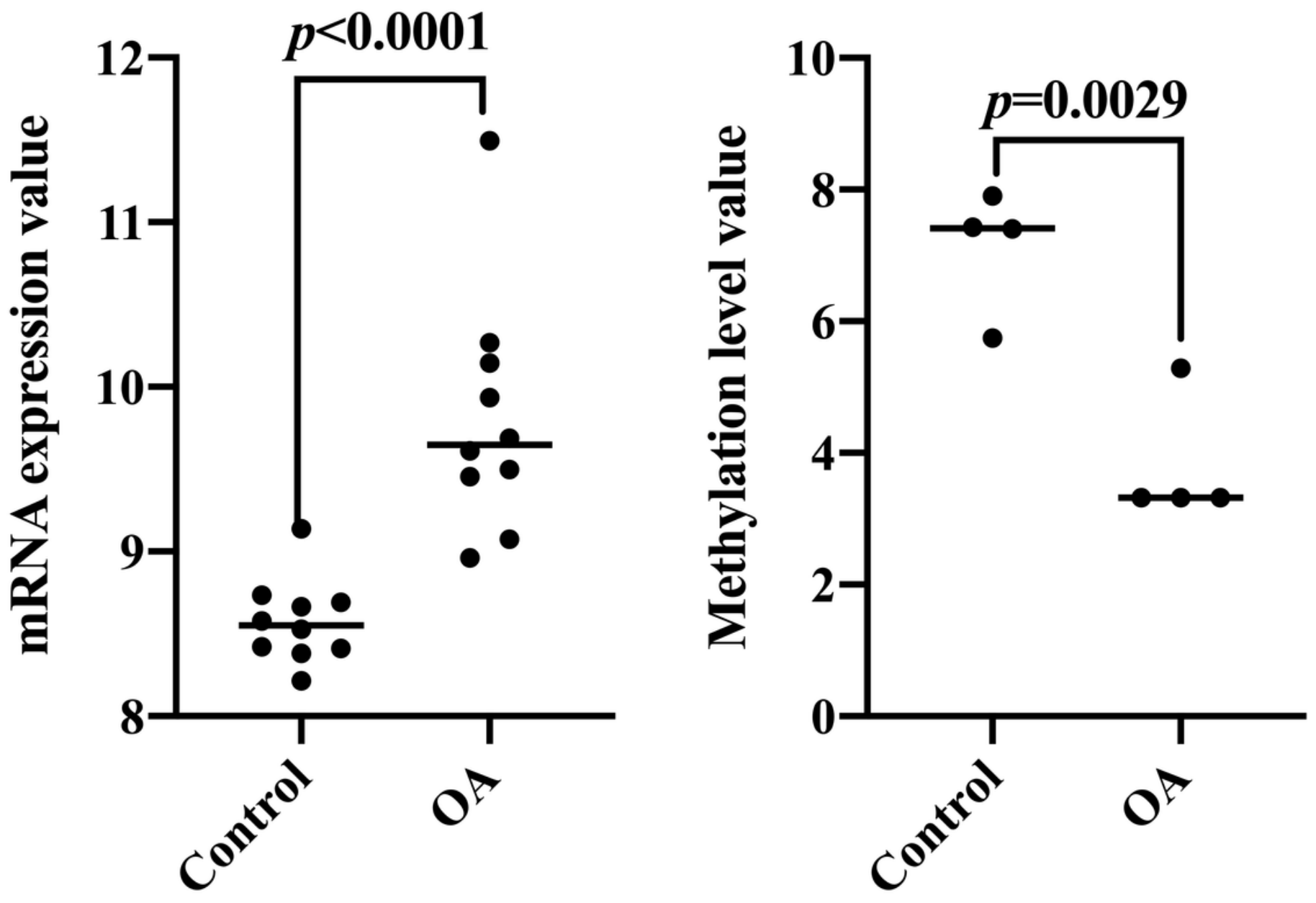

Figure 1

Transcriptome and methylation level detection in OA patients. a, Analysis of the relative expression level of CAMP mRNA in the GSE117999 dataset, ncontrol=10, nOA=10. $b$, The methylation level of CAMP gene in the GSE48422 dataset, ncontrol=4, nOA=4. 
a
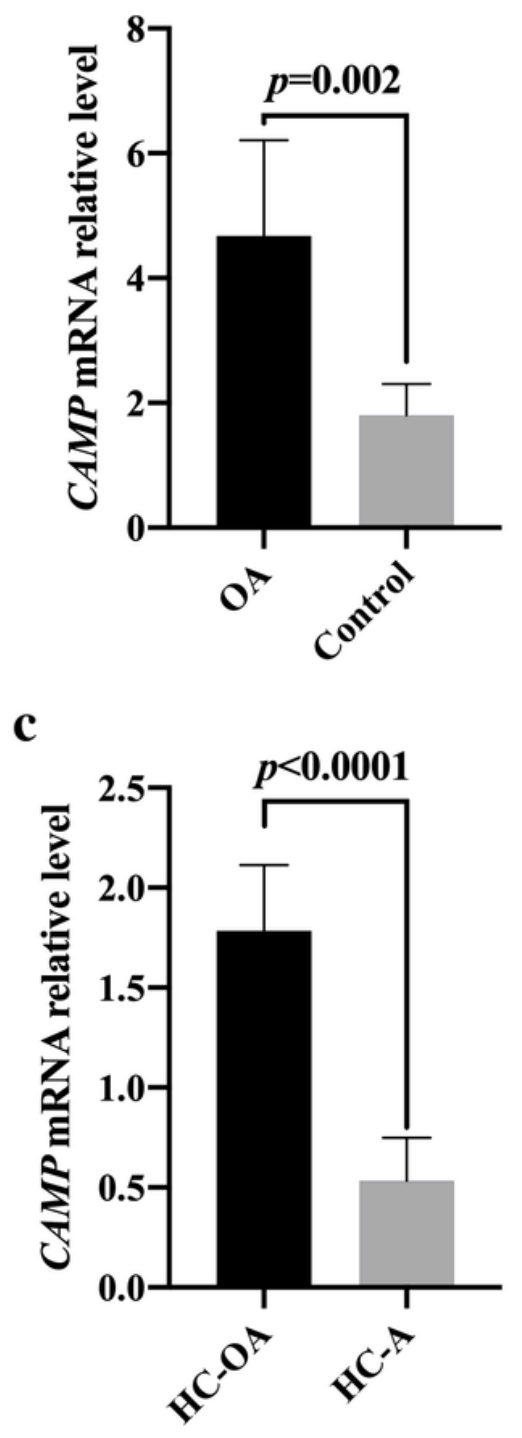

b

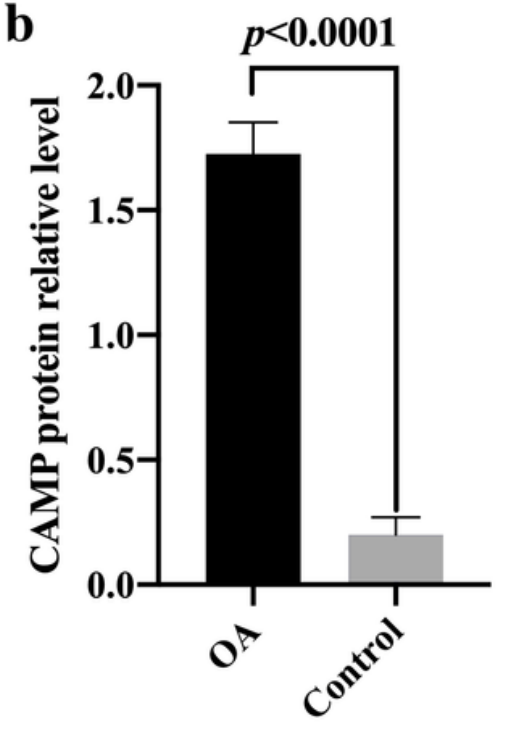

d

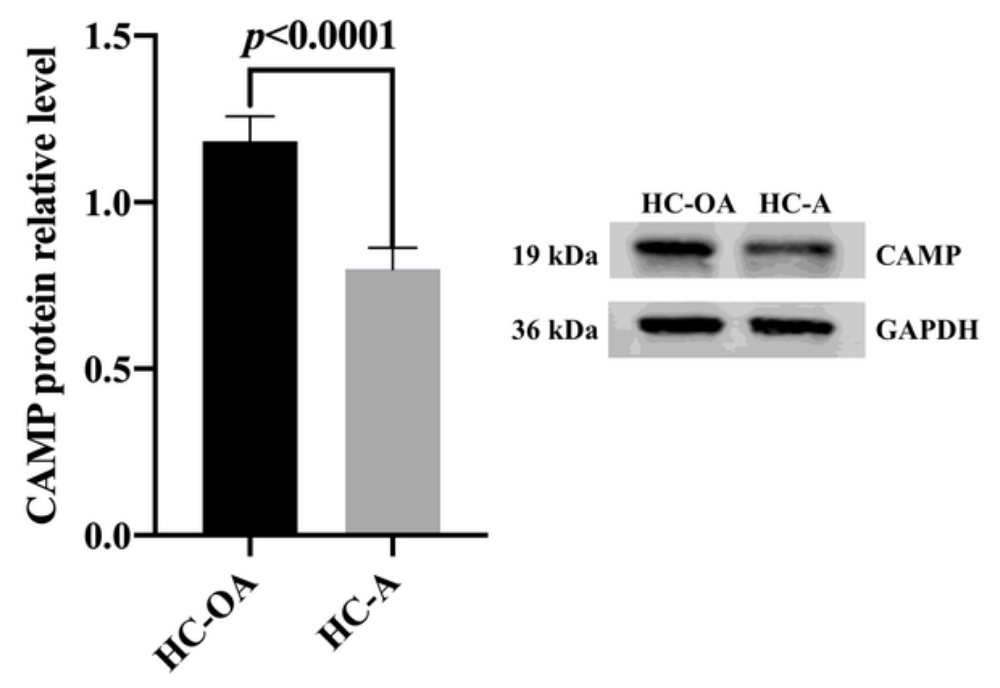

Figure 2

CAMP was highly expressed in osteoarthritis articular cartilage cells. a, The expression level of CAMP mRNA in articular cartilage tissues of OA patients and control group were analyzed by qRT-PCR; $b$, The expression level of CAMP protein in articular cartilage tissues of OA patients and control group were analyzed by Western Blot; c, CAMP mRNA expression levels in Human Chondrocytes Osteoarthritis (HC$\mathrm{OA}$ ) cells and Human Chondrocytes-articular (HC-A) cells were analyzed by qRT-PCR; d, CAMP protein expression levels in $\mathrm{HC}-\mathrm{OA}$ cells and HC-A cells were detected by Western Blot. 

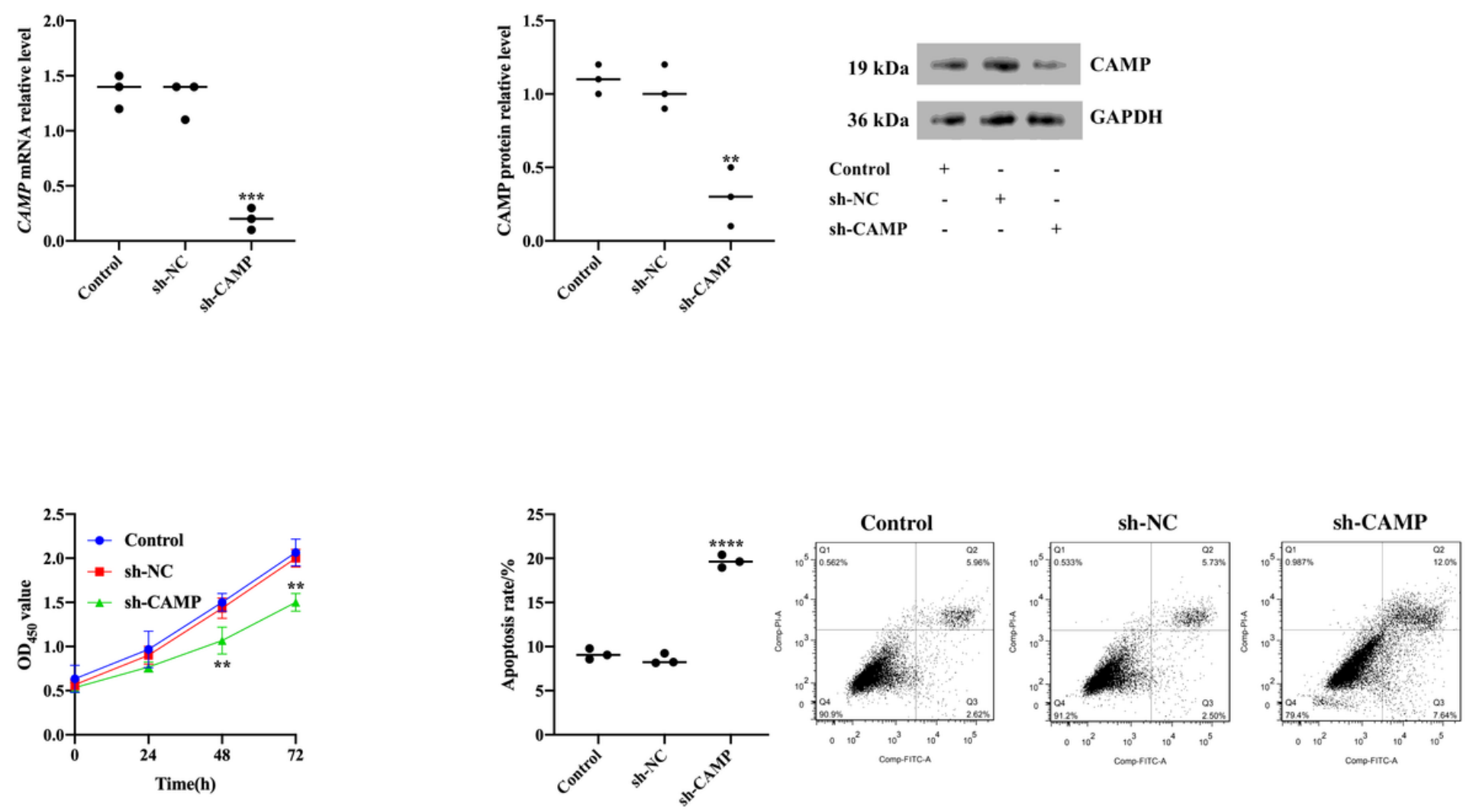

\section{Figure 3}

Silencing CAMP gene inhibited the proliferation of osteoarthritis articular cartilage cells and promoted their apoptosis. a, the expression level of CAMP mRNA in Control, sh-NC, sh-CAMP transfected HC-OA cells was detected by qRT-PCR; $b$, the expression level of CAMP protein in Control, sh-NC, sh-CAMP transfected HC-OA cell was detected by Western Blot; c, the cell proliferation ability of Control, sh-NC, shCAMP transfected HC-OA cells was detected by MTT assay; d, the apoptotic ability of Control, sh-NC, shCAMP transfected HC-OA cells was detected by Flow cytometry. ${ }^{\star *} p<0.01,{ }^{\star}{ }^{*} \mathrm{p}<<0.001,{ }^{* \star \star \star} \mathrm{p}<0.0001$, compare with Control. 

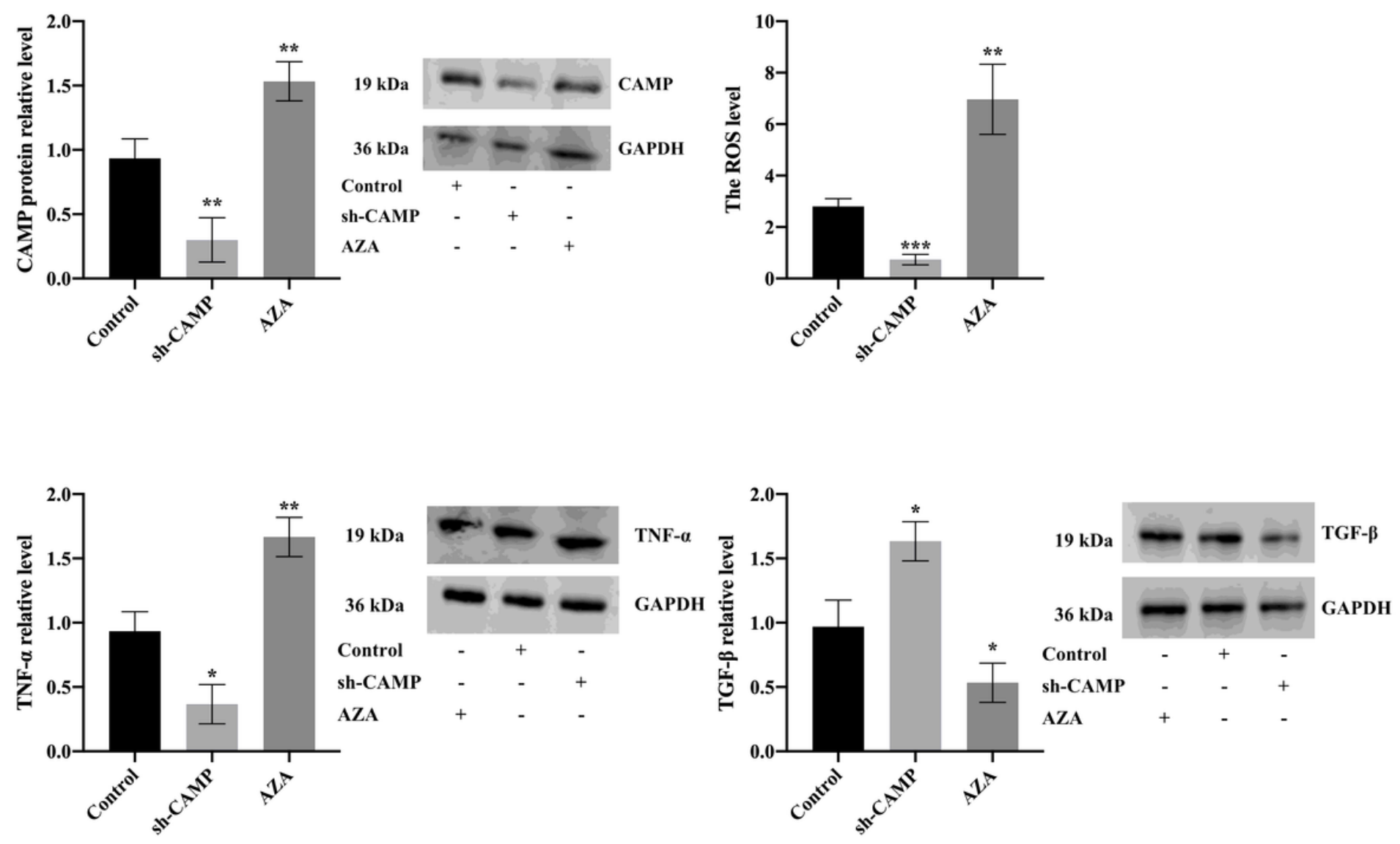

Figure 4

CAMP gene methylation inhibited ROS levels and inflammatory response levels in osteoarthritis articular cartilage cells. a, the CAMP protein expression level in Control, sh-CAMP transfected HC-OA cells and $5 \mu \mathrm{M}$ AZA treated HC-OA cells were analyzed by Western Blot; $b$, the comparison of the ROS levels in Control, sh-CAMP transfected HC-OA cells and $5 \mu \mathrm{M}$ AZA treated HC-OA cells; $\mathrm{C}$, the TNF-a protein expression levels in Control, sh-CAMP transfected HC-OA cells and $5 \mu \mathrm{M}$ AZA treated HC-OA cells were analyzed by Western Blot; $d$, the TGF- $\beta$ protein expression levels in Control, sh-CAMP transfected HC-OA cells and $5 \mu \mathrm{M}$ AZA treated HC-OA cells were analyzed by Western Blot. ${ }^{*} p<0.05$, ${ }^{* \star} p<0.01,{ }^{* \star *} p<0.001$, compared with Control. 
a

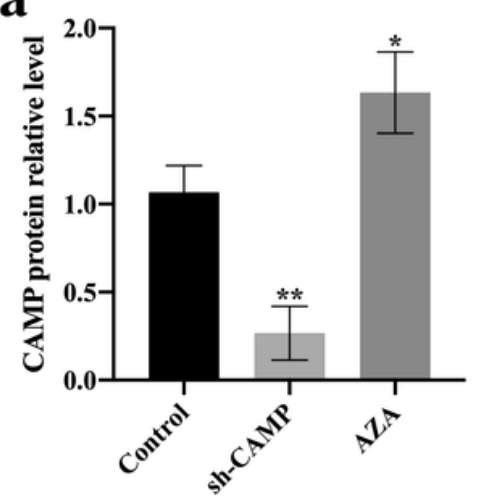

c

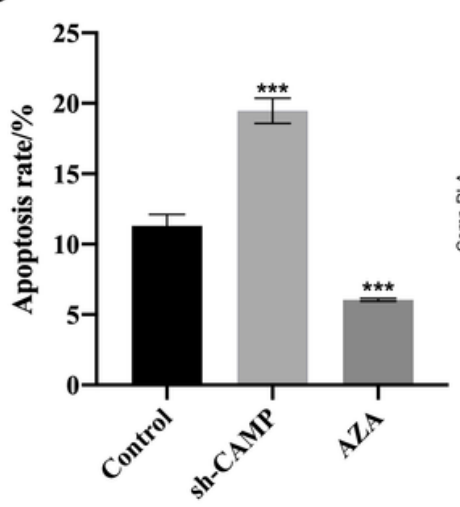

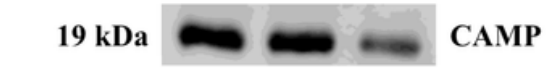

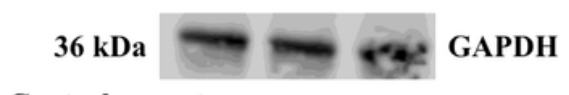

b

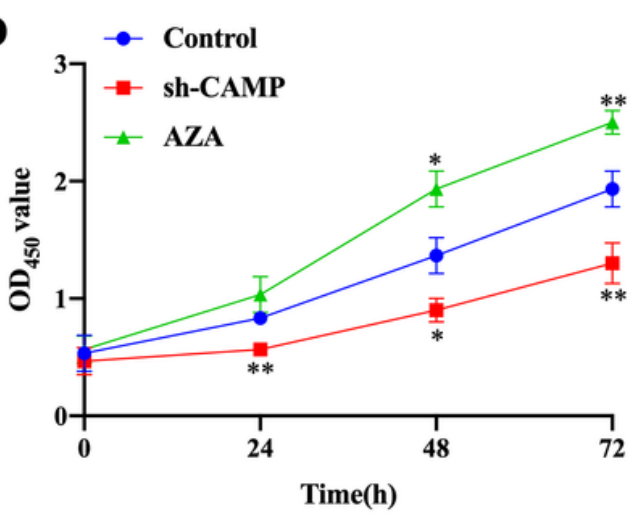

\section{Figure 5}
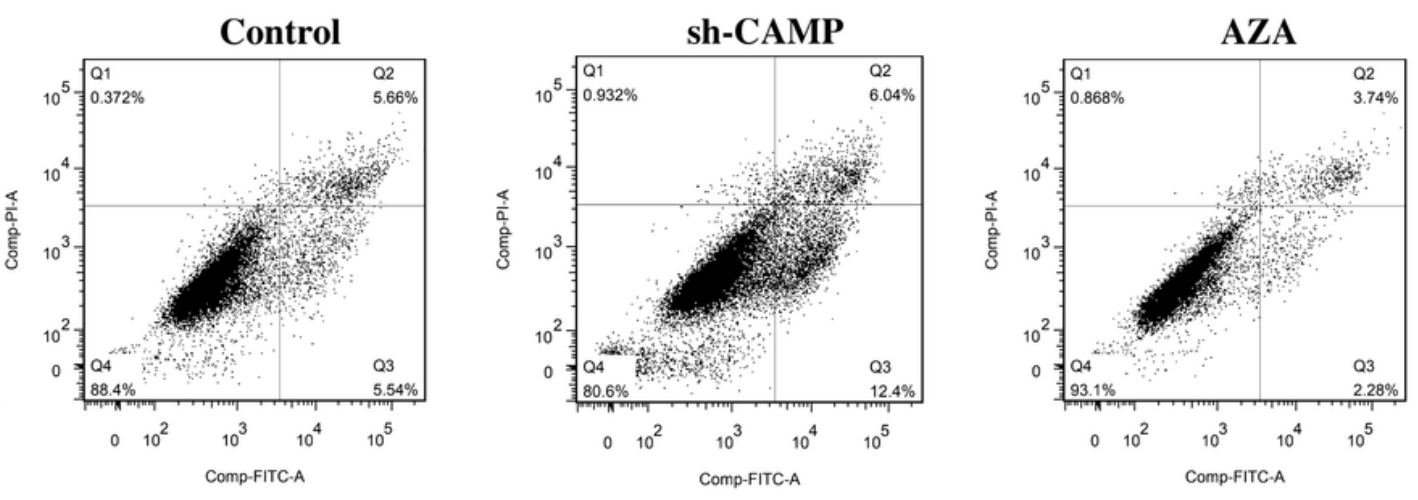

CAMP gene methylation inhibited the proliferation of osteoarthritis articular cartilage cells and promoted their apoptosis. a, the CAMP protein expression level in Control, sh-CAMP transfected HC-OA cells and $5 \mu \mathrm{M}$ AZA treated HC-OA cells were analyzed by Western Blot; $b$, the cell proliferation ability of Control, shCAMP transfected HC-OA cells and $5 \mu \mathrm{M}$ AZA treated HC-OA cells were detected by MTT assay; $c$, the apoptotic rate of Control, sh-CAMP transfected HC-OA cells and $5 \mu \mathrm{M}$ AZA treated HC-OA cells were detected by Flow cytometry. ${ }^{*} p<0.05,{ }^{* *} p<0.01,{ }^{* \star *} p<0.001$, compared with Control. 